\title{
Topological approach to Alice electrodynamics *
}

\author{
Martin Bucher, Hoi-Kwong Lo and John Preskill \\ California Institute of Technology, Pasadena, CA 91125, USA
}

Received 27 December 1991

(Revised 25 May 1992)

Accepted for publication 26 May 1992

\begin{abstract}
We analyze the unlocalized "Cheshire charge" carried by "Alice strings." The magnetic charge on a string loop is carefully defined, and the transfer of magnetic charge from a monopole to a string loop is analyzed using global topological methods. A semiclassical theory of electric charge transfer is also described.
\end{abstract}

\section{Introduction}

In a spontaneously broken non-abelian gauge theory, charge conjugation can be a local symmetry. That is, the unbroken gauge group $\mathrm{H}$ may contain both a U(1) factor generated by $Q$, and an element $X$ of the disconnected component of $\mathrm{H}$ such that $X Q X^{-1}=-Q$. Such a model contains topologically-stable cosmic strings with a remarkable property - when a charged particle is transported around the string, the sign of its charge flips. (The sign of the charge is gauge dependent, but the feature that the sign changes has an unambiguous and gauge-invariant meaning.) This string, which acts as a charge-conjugation looking glass, was first discussed by A.S. Schwarz, who dubbed it the "Alice" string [1]. (The possibility that charge conjugation could be a local symmetry was noted earlier by Kiskis [2].)

A closed loop of Alice string can carry electric charge, and the charge lost by a particle that winds around the string is transferred to the loop. A charged string loop is a peculiar object. It has a long-range electric field, from which its charge can be inferred, yet there is no localized source of charge anywhere on the string or in its vicinity [3-5]. Such charge with no locally identifiable source has been called "Cheshire charge" [4]. An Alice string can also carry magnetic Cheshire charge, and can exchange magnetic charge with magnetic monopoles $[3,6,7]$.

The properties of Alice strings that carry Cheshire charge, and the processes by which charge is exchanged between strings and point particles, have been analyzed

\footnotetext{
* This work supported in part by the US Department of Energy under Contract DE-AC03-81-ER40050.
} 
previously [4-7]. In this analysis, it is very convenient to employ the unitary gauge. However, in the presence of an Alice string, the gauge transformation that imposes the unitary gauge condition is necessarily singular; it introduces a gauge-artifact surface on which fields (the electric and magnetic fields in particular) satisfy non-trivial boundary conditions. At the price of introducing this gauge-artifact singularity, one arrives at an appealing and vivid description of the charge-transfer phenomenon.

In this paper, we analyze Cheshire charge using a different approach. In the case of magnetic charge, we note that the charge on a string is really a topological charge, and that the transfer of charge from magnetic monopole to string has an essentially topological origin. The transfer of topological charge can be described in a manifestly gauge-invariant way. By using global methods, one assuages the concern that the conclusions of previous work were an unfortunate artifact of an illicit gauge choice.

Even in the case of electric charge, global methods provide new insights. We will trace the mechanism of electric charge transfer to a generic topological property of non-abelian vortices - namely, that when one vortex winds around another, the quantum numbers of both are modified.

The rest of this paper is organized as follows: In sect. 2, we briefly review the simplest model that contains an Alice string, and recall the analysis of Cheshire charge in refs. [4-7]. In sect. 3, we describe the long-range interactions between non-abelian string loops, and use the properties of these interactions to develop a semiclassical theory of Cheshire charge and charge transfer.

In sect. 4 , we note the subtleties inherent in defining magnetic charge in the presence of loops of Alice string. For the purpose of defining the magnetic charge carried by a particular string loop, it is convenient to introduce an (arbitrary) "basepoint," and a canonical surface (or homotopy class of surfaces) that encloses the loop and is tied to the basepoint. In general, the canonical surface can be chosen in topologically inequivalent ways, and the enclosed magnetic charge depends on this choice. It is just this ambiguity that underlies the transfer of charge from a magnetic monopole to a string loop. We will find that, as a monopole winds around a string loop, the canonical surfaces that are used to define the magnetic charge of both the monopole and the loop are deformed to new (topologically inequivalent) surfaces. Therefore, the charges defined by the original canonical surfaces are modified; charge transfer has taken place.

Sect. 5 contains some concluding remarks.

\section{Alice strings}

The simplest model that contains as Alice string has gauge group SU(2) and a Higgs field $\Phi$ that transforms as the 5-dimensional irreducible representation of 
SU(2). We may express $\Phi$ as a real symmetric traceless $3 \times 3$ matrix that transforms according to

$$
\Phi \rightarrow M \Phi M^{-1}, \quad M \in \mathrm{SO}(3)
$$

If $\Phi$ has an expectation value (in unitary gauge) that can be expressed as

$$
\langle\Phi\rangle=v \operatorname{diag}[1,1,-2]
$$

then the unbroken subgroup of $\mathrm{SU}(2)$ is the semi-direct product $\mathrm{H}=\mathrm{U}(1) \times_{\text {S.D. }} \mathbb{Z}_{2}$. The unbroken group $\mathrm{H}$ has two connected components. The component connected to the identity can be pictured as rotations about a $z$-axis. Since SU(2) is a double cover of the rotation group, this component, which is isomorphic to U(1), can be expressed as $\mathrm{H}_{\mathrm{c}}=\{\exp [i \theta Q] \mid 0 \leqslant \theta<4 \pi\}$, where $Q$ is the $\mathrm{SU}(2)$ generator $Q=\frac{1}{2} \sigma_{3}$. There is also a connected component not connected to the identity of the form $\mathrm{H}_{\mathrm{d}}=\{X \exp [i \theta Q] \mid 0 \leqslant \theta<4 \pi\}$. This component consists of rotations by $180^{\circ}$ about axes that lie in the $x y$ plane. ( $X$ is any such rotation.) Each element $Y$ of $\mathrm{H}_{\mathrm{d}}$ anticommutes with $Q, Y Q Y^{-1}=-Q$; it is a "charge-conjugation" operator embedded in the unbroken local symmetry group.

The elements of $H_{d}$ represent the possible values of the "magnetic flux" of the topologically-stable cosmic string excitations of the theory in $3+1$ dimensions (or vortex excitations in $2+1$ dimensions). In general, the magnetic flux carried by a cosmic string is an element of the unbroken group $H$ that encodes the result of parallel transport along a closed path that encloses the string. To define the magnetic flux we must specify a basepoint $x_{0}$ and a closed loop $C$ that starts and ends at $x_{0}$ and encircles the string exactly once. (See fig. 1.) Then the flux is given by the untraced Wilson loop operator

$$
h\left(C, x_{0}\right)=P \exp \left(i \int_{\left(C, x_{0}\right)} \mathrm{d} x^{i} A_{i}\right)
$$

The flux takes values in $H\left(x_{0}\right)$, the subgroup of the underlying group $G$ that

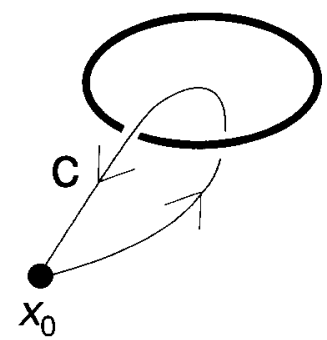

Fig. 1. The curve $C$, starting and ending at the point $x_{0}$, encloses a loop of cosmic string. 


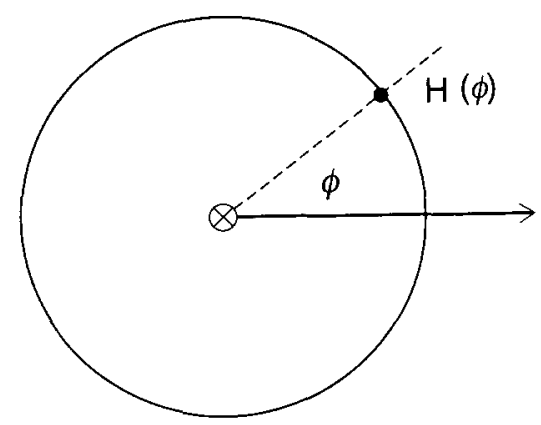

Fig. 2. A circle, parametrized by $\phi$, encloses an Alice string. Corresponding to each point of the circle is an unbroken symmetry group $H(\phi)$ that stabilizes the condensate $\Phi(\phi)$ at that point.

stabilizes the condensate at the point $x_{0}$ (since parallel transport around $C$ must return the condensate to its original value).

One can determine what happens to the charge of a particle that travels around an Alice string by considering the behavior of the unbroken symmetry group $\mathrm{H}\left(x_{0}\right)$ as it is parallel transported around the string. Consider the situation depicted in fig. 2, with a single Alice string enclosed by a circle parameterized by $\phi, 0 \leqslant \phi<2 \pi$. At each point on the circle labeled by $\phi$, there is a subgroup $\mathrm{H}(\phi)$ embedded in $\mathrm{G}$ that stabilizes the condensate $\Phi(\phi)$ at that point. The gauge vector potential $A_{\mu}$ relates these subgroups through the equation.

$$
\mathrm{H}(\phi)=U(\phi) \mathrm{H}(0) U(\phi)^{-1},
$$

where

$$
U(\phi)=P \exp \left(i \int_{0}^{\phi} \mathrm{d} \phi A_{\phi}\right) .
$$

Note that $U(2 \pi)=h\left(C, x_{0}\right)$. It is certainly true that $\mathrm{H}(0)=U(2 \pi) \mathrm{H}(0) U(2 \pi)^{-1}$, because $\mathrm{H}(2 \pi)=\mathrm{H}(0)$, but the analogous relation does not hold for the generators of $\mathrm{H}$. Since $U(2 \pi) \in \mathrm{H}_{\mathrm{d}}$, we have

$$
U(2 \pi) Q U(2 \pi)^{-1}=-Q .
$$

An analogy can be made to the Möbius strip to make it apparent why $Q$ is deformed into $-Q$ upon parallel transport around the circle. The U(1) subgroups $\{\mathrm{H}(\phi)\}$ of $\mathrm{SO}(3)$ can be represented as undirected lines in $\mathbb{R}^{3}$ through the origin that coincide with the axes of the rotation of the U(1) subgroups. Choosing a generator $Q(\phi)$ for $\mathrm{H}(\phi)$ at each $\phi$ is equivalent to choosing a direction for each of these lines. As $\phi$ varies from 0 to $2 \pi$, the lines are twisted into a Möbius strip. There is no continuous way to choose a direction on each of them.

The Möbius twist in the unbroken symmetry group $\mathrm{H}(x)$ described above may be discussed more formally in terms of the "global unrealizability" of the unbroken 
symmetry $[4,5,8]$. Let $\mathscr{M}$ denote the spatial manifold consisting of $\mathbb{R}^{3}$ with the cores of the strings excised. At each point $x \in \mathscr{M}$ is defined the unbroken symmetry group $\mathrm{H}(x)$ that stabilizes the Higgs condensate $\Phi(x)$. All these subgroups are isomorphic to the same abstract group $\mathrm{H}$. This structure is a fiber bundle $\mathrm{E}$ with model fiber $\mathrm{H}$ over the base manifold $\mathscr{M}$. The structure group of the bundle is also $\mathrm{H}$, and $\mathrm{H}$ acts on the fibers by conjugation. Locally, in any contractable open subset $\mathrm{U} \subset \mathscr{M}$, the fiber bundle has the structure $\mathrm{U} \times \mathrm{H}$. But generally there does not exist a continuous mapping

$$
f: \mathscr{M} \times \mathrm{H} \rightarrow \mathrm{E} .
$$

This is because the open sets $\mathrm{U}_{\alpha}$ covering $\mathscr{M}$ can be patched together in a non-trivial way using non-trivial transition functions. In more physical terms, a continuous mapping of the form $f$ is a "global realization" of the unbroken symmetry $\mathrm{H}$ considered as an abstract group. (In mathematical language, such a mapping is known as a trivialization of the fiber bundle E.) Clearly, such a realization is not possible in the presence of an Alice string, because such a mapping $f$ would induce a continuous choice of $Q(\phi)$ for $0 \leqslant \phi<2 \pi$, and we just showed that no such continuous choice exists. "Global unrealizability" of the unbroken symmetry also occurs when there are monopoles with non-abelian magnetic charge [9].)

The Möbius twist implies that a charged particle initially with charge $q$ will have charge $-q$ after winding around an Alice string. Of course, the sign of the charge can be changed by a gauge transformation, and therefore has no unambiguous physical meaning. But the statement that the sign changes upon transport around the string is gauge invariant and meaningful. Suppose, for example, that two charges of like sign are initially brought close together; they repel. (See fig. 3.) Then one charge travels around an Alice string while the other stays behind. When they are brought together again, they attract. Yet the total charge, as measured by an observer far away from the string loop and the point charges, cannot have changed. Where did the missing charge go?

This puzzle is resolved by Cheshire charge [4,5]. In order to understand what happened to the charge, it is convenient to choose a particular gauge - the unitary gauge in which the Higgs field takes the value eq. (2.2) everywhere. However, the gauge transformation that implements the unitary gauge condition is singular; it has a discontinuity, or cut, on a surface that is bounded by the string loop. (In other words, one can transform to unitary gauge everywhere outside a thin pancake that encloses the string loop. Inside the pancake, the Higgs field twists very rapidly, and the gauge potential is very large. The singularity arises as the width of the pancake shrinks to zero.) As a result, fields on the background of the string loop obey peculiar boundary conditions - the electromagnetic field changes sign on the cut, and charge of a charged matter field flips there. 
$+q$

$\otimes$

$+\mathrm{q}$

(a)

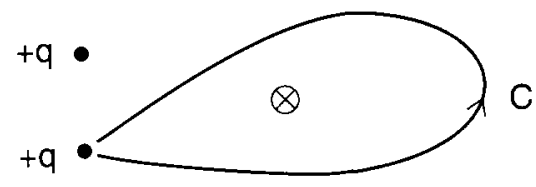

(b)

$+q$

$\otimes$

$-q$

(c)

Fig. 3. Initially two particles carry charge of the same sign. But after one of the particles travels around the string, the particles carry charge of opposite sign.

Because of the peculiar boundary conditions satisfied by the electromagnetic field at the cut, there are solutions to the classical field equations in which the cut appears to be a source of electric (or magnetic) charge, as in fig. 4. There is not actually any measurable charge density on the cut; the cut is an unphysical gauge artifact. Yet the string loop is charged - it has a long-range electric field that can be detected by a distant observer. This electric field has no locally identifiable source; it is "Cheshire charge."

The charge transfer process is sketched in fig. 5. The initial electric field of a charge- $q$ particle in the vicinity of a string loop is shown in fig. $5 \mathrm{a}$, and fig. $5 \mathrm{c}-\mathrm{e}$ shows how the field changes as the particle travels around the path in fig. $5 \mathrm{~b}$. When the particle crosses the cut, its apparent charge flips from $q$ to $-q$, and the cut seems to acquire the compensating charge $2 q$. It is clear from the final configuration in fig. 5 e that charge $2 q$ has been exchanged between the particle and the loop.

Yet there is no gauge-invariant way to pinpoint when the charge transfer took place. The configuration of the electric field lines is gauge invariant, but the direction of the arrows on the field lines is gauge dependent. We can move the cut by performing a singular gauge transformation; this alters the apparent time of the charge transfer without actually changing the physics of the process. 


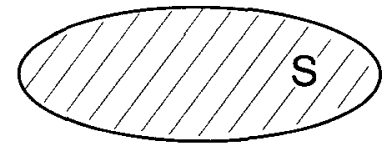

(a)

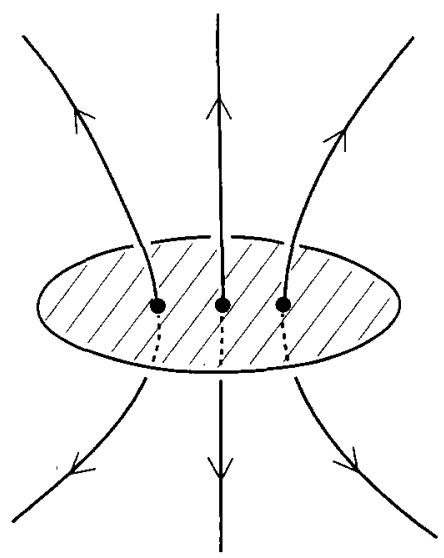

(b)

Fig. 4. The surface $S$ is a cut at which the electric field changes sign. The loop in (b) carries Cheshire charge.

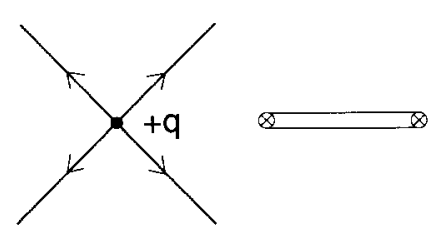

(a)

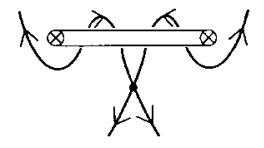

(c)

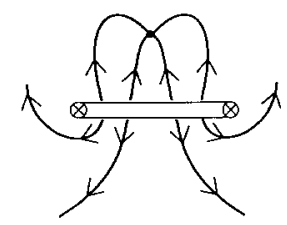

(d)

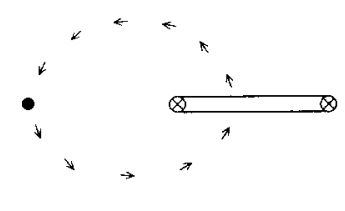

(b)

Fig. 5. A particle that initially has positive charge travels through a loop of Alice string along the path shown in (b). The electric field during the process is indicated schematically in (c)-(e). 
The charge transfer can be characterized in a gauge-invariant manner, as follows: The non-trivial irreducible representations of $\mathbf{H}$ are two-dimensional, and can be labeled by the absolute value of the U(1) charge. The tensor product of two irreducible representations decomposes into irreducible representations according to

$$
\left|q_{1}\right| \otimes\left|q_{2}\right|=\left|q_{1}+q_{2}\right| \oplus\left|q_{1}-q_{2}\right|
$$

For the charge-loop system described above, the total charge is $|q|$. This charge determines (the absolute value of) the electric flux through a large closed surface that encloses the system, and is of course conserved during the exchange process. Initially, the loop is uncharged and the particle has charge $|q|$. The exchange process leaves (the absolute value of) the charge of the particle unchanged, but produces an excitation of the loop with charge $|2 q|$.

So far, we have considered a particular model with Alice strings. Much of the physics discussed in this paper is independent of the details of that model. We will briefly describe a more general class of models in which Alice-like behavior occurs [1]. Let the unbroken group $\mathrm{H}$ be a subgroup of the simply-connected gauge group G. Topologically-stable cosmic strings occur only when $\pi_{0}(\mathrm{H})$ is non-trivial, so suppose that $\mathrm{H}$ has several connected components. Groups of this sort may be constructed as the semi-direct product of a continuous part $\mathrm{H}_{c}$, which is a connected compact Lie group, and a discrete group D. The semi-direct product $\mathrm{H}_{c} \times_{\text {S.D. }} \mathrm{D}$ is a generalization of a direct product, defined by a group homomorphism

$$
\varphi: \mathrm{D} \rightarrow \operatorname{Aut}\left[\mathrm{H}_{\mathrm{c}}\right]
$$

where $A u t\left[H_{c}\right]$ is the group of automorphisms of $H_{c}$. Group multiplication is defined using the rule

$$
\left(h_{1}, d_{1}\right) \circ\left(h_{2}, d_{2}\right)=\left(h_{1} \cdot \varphi_{d_{1}}\left(h_{2}\right), d_{1} \cdot d_{2}\right)
$$

Strings will have Alice properties if the mapping $\varphi$ is non-trivial.

In the example described earlier, $\mathrm{D}=\mathbb{Z}_{2}$ and the non-trivial automorphism reverses the sign of the generator $Q$ of $\mathrm{H}_{\mathrm{c}}=\mathrm{U}(1)$. As an example of generalized Alice behavior, consider a model with

$$
\mathrm{H}=\left[\mathrm{SU}(2)_{1} \times \mathrm{SU}(2)_{2}\right] \times \times_{\text {S.D. }} \mathbb{Z}_{2},
$$

where the non-trivial automorphism is a "parity" operator that interchanges the two SU(2) factors. (With suitable Higgs structure, the gauge group $\mathrm{G}=\mathrm{SU}(4)$ can 
be broken to this $H$.) This model contains an Alice-like string. If an object with representation content $\left(R_{1}, R_{2}\right)$ under $\mathrm{SU}(2)_{1} \times \mathrm{SU}(2)_{2}$ is transported around this string, its representation content is changed to $\left(R_{2}, R_{1}\right)$, and the missing quantum numbers are transferred to the string.

We should also note that a string might exhibit Alice-like behavior, for dynamical reasons, even when such behavior is not topologically required [4]. That is, the flux of a dynamically-stable string might assume a value $h$ that is not in the center of $\mathrm{H}$, even though there are elements of the center that lie in the same connected component as $h$. Then only the subgroup of $\mathrm{H}$ that commutes with the flux $h$ can be globally defined in the presence of the string. However, in this case, strictly speaking, the position dependence of the unbroken symmetry group $H(x)$ is not described by a topologically non-trivial bundle. This is because we can trivialize the bundle by smoothly deforming the flux $h$ to an element of the center of $\mathrm{H}$. The bundle is non-trivial only if no element of the center is contained in the same connected component as the flux; that is, only if the Alice behavior is topologically unavoidable.

\section{Electric charge}

In this section, we describe the electrically charged Alice string, and the charge transfer process, in semiclassical language [10].

In quantum theory, the electric charge of a state reflects the transformation properties of the state under global gauge transformations. The Alice string classical solution is not a charge eigenstate, but it has a "charge rotor" zero mode. Semiclassical quantization of the zero mode is achieved by constructing linear combinations of the classical string states that do have definite charge [4,5]. We need to worry, though, about what is meant by a "global" gauge transformation, since we have seen that gauge transformations are not globally realizable. Fortunately, for the purpose of defining the total charge of a state, it is sufficient to consider a gauge transformation that is constant on and outside a large sphere that encloses all of the charged objects. Inside the sphere, we may deform the gauge transformation so that it vanishes on the core of each string, and on a surface bounded by each string [5]. There is no topological obstruction to constructing this gauge transformation. Strictly speaking, since the flux of a string is defined relative to a basepoint, we should think of the large sphere not as a "free" surface, but rather as a surface tied to the basepoint $x_{0}$. That is, the gauge transformation takes the same value at $x_{0}$ as on the sphere. (If the total magnetic charge enclosed by the sphere is non-zero, then there is a further obstruction, so that the gauge transformations in the disconnected component $\mathrm{H}_{\mathrm{d}}$ cannot be defined on the sphere [9]. We defer the discussion of magnetically charged string loops until sect. 4 , and suppose, for now, that the magnetic charge is zero.) 
The magnetic flux of the string, defined by eq. (2.3), takes values in the disconnected component $\mathrm{H}_{\mathrm{d}}$ of the unbroken group $\mathrm{H}\left(x_{0}\right)$ that stabilizes the condensate at the basepoint $x_{0}$. In general, this flux transforms under a transformation $g \in \mathrm{H}\left(x_{0}\right)$ according to

$$
h\left(C, x_{0}\right) \rightarrow g h\left(C, x_{0}\right) g^{-1} .
$$

In the case of an Alice string, let $|\theta\rangle$ denote the string loop state with flux $h(C$, $\left.x_{0}\right)=X \mathrm{e}^{i \theta Q}$. Under a global H-transformation, the transformation property (3.1) becomes

$$
\begin{aligned}
U\left(\mathrm{e}^{i \omega Q}\right)|\theta\rangle & =|\theta-2 \omega\rangle, \\
U\left(X \mathrm{e}^{i \omega Q}\right)|\theta\rangle & =|2 \omega-\theta\rangle,
\end{aligned}
$$

where $U$ is the unitary operator acting on Hilbert space that represents the global gauge transformation.

One can construct linear combinations of these "flux eigenstate" string states that transform irreducibly under $\mathrm{H}$. Let

$$
|q\rangle=\int_{0}^{4 \pi} \frac{\mathrm{d} \theta}{\sqrt{4 \pi}} \mathrm{e}^{i(\theta / 2) q}|\theta\rangle
$$

(where $q$ is an integer). It transforms as

$$
\begin{gathered}
U\left(\mathrm{e}^{i \omega Q}\right)|q\rangle=\mathrm{e}^{i \omega q}|q\rangle, \\
U\left(X \mathrm{e}^{i \omega Q}\right)|q\rangle=\mathrm{e}^{i \omega q}|-q\rangle .
\end{gathered}
$$

The two states $|q\rangle$ and $|-q\rangle$ thus comprise the basis for an irreducible representation of $\mathrm{H}$.

Only integer- $|q|$ representations of $\mathrm{H}$ occur in this decomposition; an Alice string cannot carry half-integer $|q|$. String loops are invariant under the center of SU(2), and so can have no "two-ality."

The semiclassical quantization of the charge rotor of the Alice string is strongly reminiscent of the corresponding treatment of bosonic superconducting strings [11]. But the physical properties of the string are actually remarkably different. Alice strings do not carry persistent currents. Instead, they carry electric charge (or magnetic charge, as we will discuss in sect. 4).

Now we will discuss the charge transfer process. It will be enlightening to imagine that the charge object that winds through the string loop is itself a loop of Alice string. Then the charge transfer can be regarded as a consequence of a topological interaction between non-abelian string loops. (We will see in sect. 4 that magnetic charge transfer results from a related topological interaction.) 


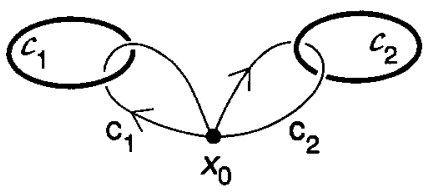

(a)

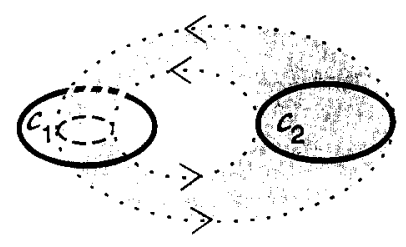

(b)

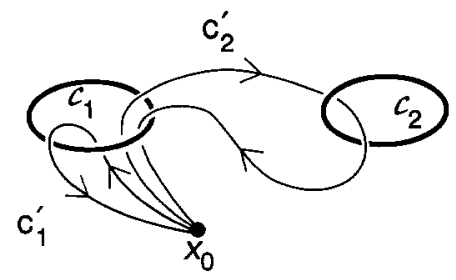

(c)

Fig. 6. The flux on the two string loops $\mathscr{C}_{1}$ and $\mathscr{E}_{2}$ is defined with respect to the basepoint $x_{0}$ and the paths $C_{1}$ and $C_{2}$. When $\mathscr{C}_{2}$ winds through $\mathscr{E}_{1}$ as in (b), the paths $C_{1}^{\prime}$ and $C_{2}^{\prime}$ are dragged to $C_{1}$ and $C_{2}$.

Consider the system of two string loops $\mathscr{C}_{1}$ and $\mathscr{C}_{2}$ shown in fig. 6a. Suppose that each string is a flux eigenstate, with

$$
\begin{aligned}
& h\left(C_{1}, x_{0}\right)=h_{1}, \\
& h\left(C_{2}, x_{0}\right)=h_{2} .
\end{aligned}
$$

Now suppose that the loop $\mathscr{C}_{2}$ winds through $\mathscr{C}_{1}$ as in fig. 6 b. To determine the magnetic flux of the loops after the winding, it is convenient to consider the paths $C_{1}^{\prime}$ and $C_{2}^{\prime}$ in fig. 6c. During the winding procedure, these paths are dragged back to the paths $C_{1}$ and $C_{2}$. Therefore, the flux associated with the paths $C_{1}$ and $C_{2}$ after the winding is the same as the flux associated with the paths $C_{1}^{\prime}$ and $C_{2}^{\prime}$ before the winding. One sees that $C_{1}^{\prime}=C_{1}$ and $C_{2}^{\prime}=C_{1}^{-1} \circ C_{2} \circ C_{1}$. (Our convention is that $C_{2} \circ C_{1}$ denotes the path that is obtained by traversing first $C_{1}$, then 
$C_{2}$.) We therefore find that, after the winding, the flux carried by the string loops is [12-14].

$$
\begin{aligned}
& h^{\prime}\left(C_{1}, x_{0}\right)=h\left(C_{1}^{\prime}, x_{0}\right)=h_{1}, \\
& h^{\prime}\left(C_{2}, x_{0}\right)=h\left(C_{2}^{\prime}, x_{0}\right)=h_{1}^{-1} h_{2} h_{1} .
\end{aligned}
$$

In the case of Alice strings, we denote by $\left|\theta_{1}, \theta_{2}\right\rangle$ the two-string state with flux $h_{1}=X \mathrm{e}^{i \theta_{1} Q}$ and $h_{2}=X \mathrm{e}^{i \theta_{2} Q}$. Then, if string 2 winds through string 1 , eq. (3.8) becomes

$$
\left|\theta_{1}, \theta_{2}\right\rangle \rightarrow\left|\theta_{1}, 2 \theta_{1}-\theta_{2}\right\rangle
$$

If we construct charge eigenstates as in eq. (3.4), we find from eq. (3.9) that the effect of the winding is

$$
\left|\theta_{1}, q_{2}\right\rangle \rightarrow \mathrm{e}^{i \theta_{1} q_{2}}\left|\theta_{1}-q_{2}\right\rangle
$$

and

$$
\left|q_{1}, q_{2}\right\rangle \rightarrow\left|q_{1}+2 q_{2},-q_{2}\right\rangle
$$

Just as in the classical analysis of sect. 2, the sign of $q_{2}$ flips, and loop 1 acquires a compensating charge.

Of course, we can also analyze (somewhat more straightforwardly) the case in which the charge that winds is a point charge rather than a charged loop. Then eq. (3.10) follows directly from the gauge transformation property of the charged particle.

\section{Magnetic charge}

\subsection{TWISTED FLUX}

In the above discussion of semiclassical quantization, we assumed that the magnetic flux was a constant along the string. But if the unbroken group $\mathrm{H}$ is continuous as in the Alice case, the flux can vary as a function of position along the string loop. Furthermore, if $\mathrm{H}$ is not simply connected, then the flux might trace out a non-contractible closed path in $\mathrm{H}$. Then the string loop evidently carries a type of topological charge. This charge is precisely the magnetic charge of the loop.

To define this charge carefully, we should, as usual, select an arbitrary basepoint $x_{0}$ and consider the magnetic flux defined by eq. (2.3). As the path $C$ is smoothly deformed with the basepoint $x_{0}$ held fixed, this flux varies smoothly in a given connected component of the group $\mathrm{H}\left(x_{0}\right)$. To be specific, consider the family 


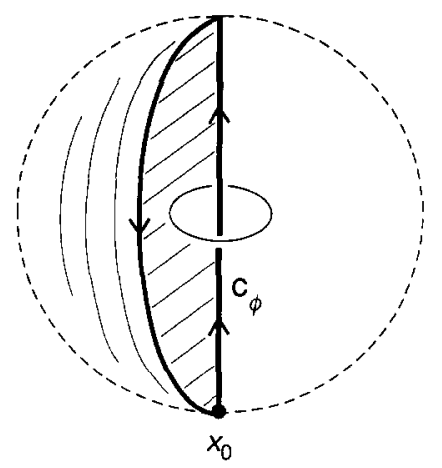

Fig. 7. The family of closed paths $\left\{C_{\phi} \mid 0 \leqslant \phi<2 \pi\right\}$ sweeps out a degenerate torus that encloses the Alice string loop.

of paths $\left\{C_{\phi} \mid 0 \leqslant \phi<2 \pi\right\}$, shown in fig. 7. These paths sweep out a degenerate torus that encloses the string loop. This family $\left\{C_{\phi}\right\}$ is associated with a closed path in $\mathrm{H}\left(x_{0}\right)$ that begins and ends at the identity; namely,

$$
h\left(C_{\phi}, x_{0}\right) h^{-1}\left(C_{\phi=0}, x_{0}\right), \quad 0 \leqslant \phi<2 \pi .
$$

We have thus found a natural way of mapping a two-sphere that encloses the string loop to a closed loop in $\mathrm{H}_{\mathrm{c}}$, the component of $\mathrm{H}$ connected to the identity.

By smoothly deforming the family $\left\{C_{\phi}\right\}$, we may obtain the family of closed paths $\left\{C_{\phi}^{\prime}\right\}$ shown in fig. 8. Loosely speaking, $h\left(C_{\phi}^{\prime}, x_{0}\right)$ is the flux carried by the string at the point where $C_{\phi}^{\prime}$ wraps around the core of the string. Thus we see that the homotopy class of the path defined by eq. (4.1) describes how the flux of the string twists as a function of position along the string.

On the other hand, the family $\left\{C_{\phi} C_{\phi=0}^{-1}\right\}$ is equivalent to the family of paths $\left\{C_{\phi}^{\prime \prime}\right\}$ shown in fig. 9. But this is just the family of paths used by Lubkin $[6,15,16]$ to define the topological $\mathrm{H}_{c}$ magnetic charge inside a two-sphere. We learn that the element of $\pi_{1}\left(\mathrm{H}_{\mathrm{c}}\right)$ that characterizes how the magnetic flux of the string twists is the same as the magnetic charge on the loop $[10,6]$.

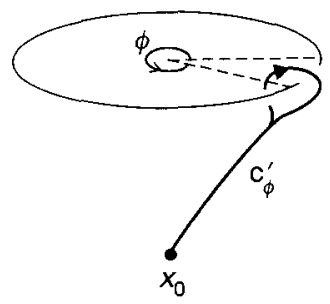

Fig. 8. A family of closed paths $\left\{C_{\phi}^{\prime}\right\}$ obtained by smoothly deforming the family $\left\{C_{\phi}\right\}$. 


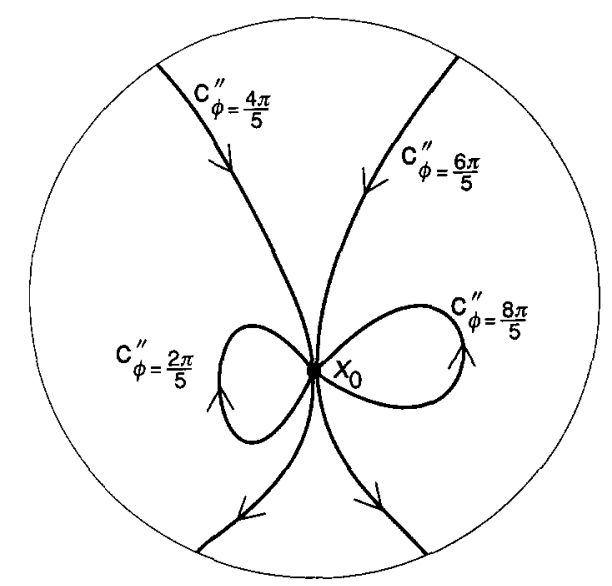

Fig. 9. A family of loops $C_{\phi}^{\prime \prime}$ that sweeps over the surface of a sphere. The loops $C_{0}^{\prime \prime}$ and $C_{2 \pi}^{\prime \prime}$ are degenerate.

More generally, in the presence of many string loops and pointlike monopoles, we can define the magnetic charge inside any region $\mathbf{R}$ whose boundary $\partial \mathbf{R}$ is homeomorphic to $\mathrm{S}^{2}$. The result is a homomorphism

$$
h^{(2)}: \pi_{2}\left[\mathscr{M}, x_{0}\right] \rightarrow \pi_{1}\left[\mathrm{H}_{\mathrm{c}}\left(x_{0}\right)\right]
$$

where $\mathscr{M}$ denotes the manifold that is obtained when all string loops and monopoles are removed from $\mathbb{R}^{3}$.

\subsection{ROLE OF THE BASEPOINT}

We should now explain why it is important to specify a basepoint $x_{0}$ for the purpose of defining the magnetic charge. Naively, it seems that it should be possible to define the magnetic charge enclosed by a "free" surface that is not tied to any basepoint, since the enclosed charge is just the magnetic flux through the surface. But trouble arises if we allow the magnetic charges to move. We can deform the free surface so that it is never crossed by any moving magnetic monopoles or string loops. Nevertheless, the magnetic flux through the surface can change if the surface winds through an Alice string loop.

It will be easier to keep track of charge transfer processes if we define magnetic charge using a surface that is tied to a basepoint. As the charges move, we can again deform the surface so that no monopoles or strings cross it, while keeping the basepoint fixed (as long as no monopoles or strings cross the basepoint). Then the magnetic charge enclosed by the surface remains invariant. However, when a monopole winds around a string loop, the surface enclosing the monopole becomes deformed to a new, topologically inequivalent surface. We can then find how the 


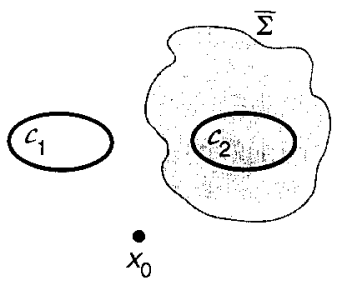

(a)

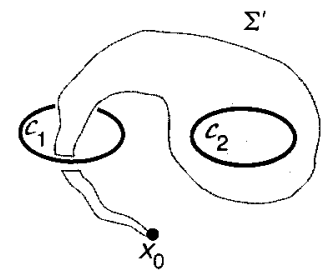

(c)

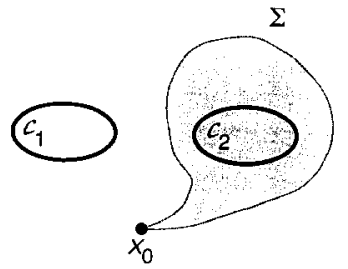

(b)

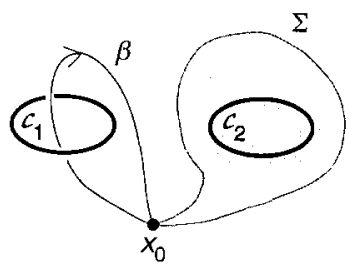

(d)

Fig. 10. The free surface $\bar{\Sigma}$ in (a) can be threaded to the basepoint $x_{0}$ in inequivalent ways, two of which are illustrated in (b) and (c). The surface (c) can be deformed to (d), which differs from (b) by the degenerate tube $\beta$ that begins and ends at the basepoint.

charge of the monopole has changed by expressing the new surface in terms of the old one. This procedure is closely analogous to our discussion in sect. 3 of how the flux of a loop is modified when it winds around another string. There we defined the flux using a standard path that became deformed to a new path due to the winding. We can analyze the exchange of magnetic charge using a similar strategy, except that a surface, rather than a path, is used to define the charge.

In order to define the magnetic charge enclosed by a free surface $\bar{\Sigma}$ that is homeomorphic to $S^{2}$, then, we specify not just the surface, but also a path that attaches the surface to the basepoint $x_{0}$. Of course, this path can be chosen in many topologically inequivalent ways; the different choices are classified by $\pi_{1}[\mathscr{M}$, $\left.x_{0}\right]$. Thus, $\pi_{1}\left[\mathscr{H}, x_{0}\right]$ classifies the ambiguity in associating a free surface with an element of $\pi_{2}\left[\mathscr{M}, x_{0}\right]$. There is a corresponding ambiguity in the value of the magnetic charge (given by the homomorphism $h^{(2)}$ defined in eq. (4.2)) that is associated with a free surface. We resolve this ambiguity by simply choosing a standard convention for the path from the free surface to the basepoint, and sticking with this convention throughout the process under study.

The ambiguity is illustrated by fig. 10 , which shows two inequivalent surfaces $\Sigma$ and $\Sigma^{\prime}$ with basepoint $x_{0}$ that are obtained by "threading" the free surface $\bar{\Sigma}$ to the basepoint in two different ways. As shown in fig. $10 \mathrm{~d}$, the surface $\Sigma$ ' can be deformed to a degenerate tube, beginning and ending at $x_{0}$, joined to the surface $\Sigma$. Since the degenerate tube is equivalent to a closed path $\beta$, we may say that the two surfaces differ by an element of $\pi_{1}\left[\mathscr{M}, x_{0}\right]$. 
The ambiguity in associating a free surface with an element of $\pi_{2}\left[\mathscr{M}, x_{0}\right]$ can be characterized by a natural homomorphism

$$
\tau: \pi_{1}\left[\mathscr{M}, x_{0}\right] \rightarrow \operatorname{Aut}\left(\pi_{2}\left[\mathscr{M}, x_{0}\right]\right)
$$

that takes (homotopy classes of) closed paths to automorphisms of $\pi_{2}\left[\mathscr{M}, x_{0}\right]$. The mapping $\tau$ is defined in the following way: Let $\beta \in \pi_{1}\left[\mathscr{M}, x_{0}\right]$ and $\Sigma \in \pi_{2}\left[\mathscr{M}, x_{0}\right]$. (Below we use the symbols $\beta$ and $\Sigma$ to denote both homotopy equivalence classes and particular representatives of the classes.) Then $\tau_{\beta}$ is an automorphism that takes $\Sigma$ to a new surface $\Sigma^{\prime}$,

$$
\tau_{\beta}: \Sigma \rightarrow \Sigma^{\prime}
$$

where $\Sigma^{\prime}$ is the surface $\Sigma$ with the degenerate tube $\beta$ added on. More precisely, let $\beta(t), 0 \leqslant t \leqslant 1$ be a parametrized path, with $\beta(0)=\beta(1)=x_{0}$, and let $\Sigma(\theta, \phi)$, $0 \leqslant \theta \leqslant \pi, 0 \leqslant \phi \leqslant 2 \pi$ be a parametrized surface with $\Sigma(0, \phi)=x_{0}$. Then the new surface $\Sigma^{\prime}$ is

$$
\Sigma^{\prime}(\theta, \phi)=\left\{\begin{array}{lll}
\beta(2 \theta / \pi) & \text { if } & 0 \leqslant \theta \leqslant \pi / 2 \\
\Sigma(2 \theta-\pi, \phi) & \text { if } & \pi / 2 \leqslant \theta \leqslant \pi .
\end{array}\right.
$$

Now consider how changing the threading of a free surface to the basepoint modifies the magnetic charge enclosed by the surface. Recall that eq. (2.3) maps a path that begins and ends at the basepoint to an element of the group $\mathrm{H}\left(x_{0}\right)$. If the path is deformed to a homotopically equivalent path, the group element remains in the same connected component of the group. Thus, eq. (2.3) defines a homomorphism

$$
h^{(1)}: \pi_{1}\left[\mathscr{M}, x_{0}\right] \rightarrow \pi_{0}\left[H\left(x_{0}\right)\right] .
$$

If the surface $\Sigma$ is changed to the surface $\Sigma^{\prime}$ by adding the degenerate tube $\beta$, then the magnetic charge enclosed by the new surface is related to the magnetic charge enclosed by the original surface according to

$$
h^{(2)}\left(\Sigma^{\prime}\right)=h^{(1)}(\beta)^{-1} h^{(2)}(\Sigma) h^{(1)}(\beta) .
$$

That is, $h^{(2)}\left(\Sigma^{\prime}\right)$ is the closed path in $\mathrm{H}_{\mathrm{c}}$ (beginning and ending at the identity) that is obtained when $h^{1}(\beta)$ acts on the closed path $h^{(2)}(\Sigma)$ by conjugation. In the case of the Alice string, eq. (4.7) simply says that, if $\beta$ is a path that winds around a string loop, then the magnetic charges enclosed by $\Sigma$ and $\Sigma^{\prime}$ differ by a sign.

\subsection{CHARGE TRANSFER}

Eq. (4.7) is the key to understanding the magnetic charge transfer process, as we will show. First, through, we should recall that $\pi_{2}\left[\mathscr{M}, x_{0}\right]$ has a group structure 


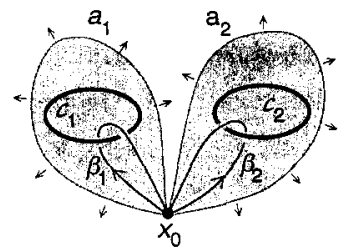

(a)

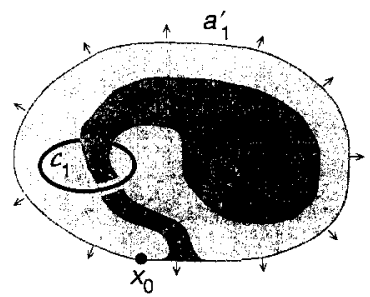

(c)

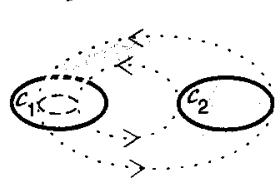

(b)

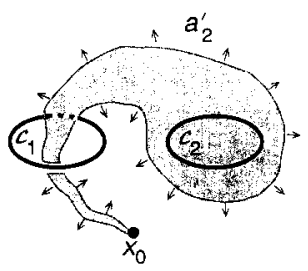

(d)

Fig. 11. The magnetic flux of the string loops $\mathscr{C}_{1}$ and $\mathscr{E}_{2}$ is defined in terms of the paths $\beta_{1}$ and $\beta_{2}$ shown in (a), and the magnetic charges of the loops are defined in terms of the surfaces $a_{1}$ and $a_{2}$; the paths and the surfaces are based at the point $x_{0}$. When $\mathscr{E}_{2}$ winds through $\mathscr{C}_{1}$ as in (b), the surface $a_{1}^{\prime}$ shown in (c) is dragged to $a_{1}$, and the surface $a_{2}^{\prime}$ shown in (d) is dragged to $a_{2}$. The arrows on the surfaces indicate outward-pointing normals.

that allows magnetic charge to be added. The group multiplication law,

$$
\circ: \pi_{2}\left[\mathscr{M}, x_{0}\right] \times \pi_{2}\left[\mathscr{M}, x_{0}\right] \rightarrow \pi_{2}\left[\mathscr{M}, x_{0}\right]
$$

can be defined as

$$
\left(\Sigma_{1} \circ \Sigma_{2}\right)(\theta, \phi)=\left\{\begin{array}{lll}
\Sigma_{1}(2 \theta, \phi) & \text { if } & 0 \leqslant \theta \leqslant \pi / 2 \\
\Sigma_{2}(2 \theta-\pi, \phi) & \text { if } & \pi / 2 \leqslant \theta \leqslant \pi .
\end{array}\right.
$$

where $\Sigma_{1}, \Sigma_{2}$, and $\Sigma_{1} \circ \Sigma_{2}$ are homotopy equivalence class representatives. Group multiplication in $\pi_{2}$ is commutative. Group inversion may be expressed in terms of class representatives as

$$
\Sigma^{-1}(\theta, \phi)=\Sigma(\pi-\theta, \phi)
$$

We turn to the situation depicted in fig. 11. Two string loops $\mathscr{C}_{1}$ and $\mathscr{C}_{2}$ are shown. We denote by $\beta_{1}$ and $\beta_{2}$ two standard paths, beginning and ending at the basepoint $x_{0}$, that wind around the string loops. (These are elements of $\pi_{1}[\mathscr{M}$, $x_{0}$ ].) We denote by $a_{1}$ and $a_{2}$ two standard surfaces, based at $x_{0}$, that enclose the string loops. (These are elements of $\pi_{2}\left[\mathscr{M}, x_{0}\right]$.) The magnetic charges of the two loops, given by the homomorphism (4.2), are $h^{(2)}\left(a_{1}\right)$ and $h^{(2)}\left(a_{2}\right)$, respectively. 


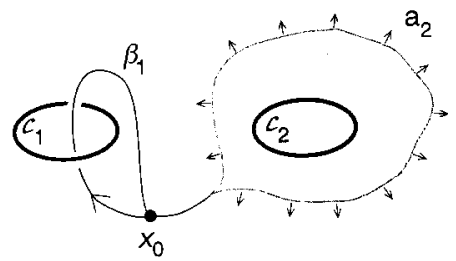

(a)

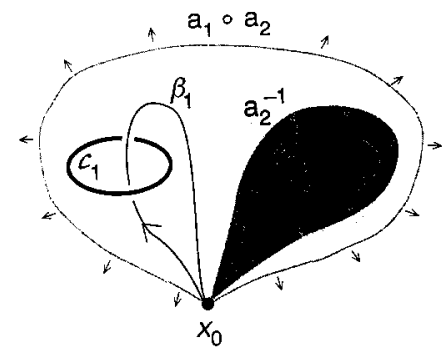

(b)

Fig. 12. Deformations of the surfaces shown in fig. 11c-d. In (a), the surface $a_{2}^{\prime}$ has been deformed to the degenerate tube $\beta_{1}$ plus the surface $a_{2}$. In (b), the surface $a_{1}^{\prime}$ has been deformed to the surface $a_{1} \circ a_{2}$ that encloses both loops, plus the inverse of $a_{2}^{\prime}$ (that is, $a_{2}^{\prime}$ with the orientation reversed); the surface $\left(a_{2}^{\prime}\right)^{-1}$ is the sum of the degenerate tube $\left(\beta_{1}\right)^{-1}$ and the surface $\left(a_{2}\right)^{-1}$.

Now suppose that the loop $\mathscr{C}_{2}$ winds through the loop $\mathscr{C}_{1}$ along the path shown in fig. 11b. We want to determine the magnetic charges of the two loops after this winding. To do so, consider the surfaces $a_{1}^{\prime}$ and $a_{2}^{\prime}$ shown in fig. 11c-d. During the winding, these surfaces are dragged back to the surfaces $a_{1}$ and $a_{2}$, if the surfaces are deformed so that no surface ever touches a string loop. Therefore, the magnetic charge enclosed by $a_{1}$, after the winding, is the same as the magnetic charge enclosed by $a_{1}^{\prime}$, before the winding. Similarly, the magnetic charge enclosed by $a_{2}$, after the winding, is the same as the magnetic charge enclosed by $a_{2}^{\prime}$, before the winding.

It remains to find the magnetic charges enclosed by $a_{1}^{\prime}$ and $a_{2}^{\prime}$ before the winding. Fig. 12a shows a deformation of $a_{2}^{\prime}$ that makes it manifest that $a_{2}^{\prime}$ can be expressed as

$$
a_{2}^{\prime}=\tau_{\beta_{1}}\left(a_{2}\right),
$$

where $\tau_{\beta_{1}}$ is the automorphism of $\pi_{2}\left[\mathscr{M}, x_{0}\right]$ defined by eq. (4.4)-(4.5). In fig. 12b, the surface $a_{1}^{\prime}$ is expressed as the sum of two surfaces. The first (outer) surface is just $a_{1} \circ a_{2}$, the surface that encloses both loops. The second (inner) surface is $\left(a_{2}^{\prime}\right)^{-1}$; it is the same as $a_{2}^{\prime}$, except with the opposite orientation. We see that

$$
a_{1}^{\prime}=a_{1} \circ a_{2} \circ\left(a_{2}^{\prime}\right)^{-1} \text {. }
$$


Finally, we apply eq. (4.7) to find the magnetic charges after the winding; the result is

$$
\begin{aligned}
& h^{(2)^{\prime}}\left(a_{1}\right)=h^{(2)}\left(a_{1}^{\prime}\right)=h^{(2)}\left(a_{1} \circ a_{2}\right)\left[h^{(2)^{\prime}}\left(a_{2}\right)\right]^{-1} \\
& h^{(2)^{\prime}}\left(a_{2}\right)=h^{(2)}\left(a_{2}^{\prime}\right)=h^{(1)}\left(\beta_{1}\right)^{-1} h^{(2)}\left(a_{2}\right) h^{(1)}\left(\beta_{1}\right) .
\end{aligned}
$$

Of course, the total magnetic charge is unchanged, because $h^{(2)}\left(a_{1} \circ a_{2}\right)=$ $h^{(2)}\left(a_{1} \circ a_{2}\right)$.

In the case of the Alice string, the magnetic charge can be labeled by an integer $p$ - the charge in units of the Dirac charge. If the magnetic charges on the string loops are initially $p_{1}$ and $p_{2}$, and loop 2 winds through loop 1, eq. (4.13) says that the charges become modified according to

$$
\left|p_{1}, p_{2}\right\rangle \rightarrow\left|p_{1}+2 p_{2},-p_{2}\right\rangle
$$

in accord with the analysis in sect. 2.

\subsection{DYONS}

We may also consider dyonic Alice string loops, that carry both magnetic and electric charge. The classical magnetically charged Alice string loop has a charge rotor zero-mode, just like the magnetically neutral loop considered in sect. 3, and we may proceed with semiclassical quantization in the same manner as before. The only difference from the previous discussion is that, for the magnetically charged loop, there is a topological obstruction to defining global gauge transformations in the disconnected component of the unbroken group $\mathrm{H}$, similar to the obstruction discussed in ref. [9]. (The obstruction occurs because the automorphism that reverses the sign of $Q$ is incompatible with the matching condition of a magnetic monopole.) Thus, we obtain states that transform irreducibly under the connected component $\mathrm{H}_{\mathrm{c}}=\mathrm{U}(1)$, but the states do not transform as representations of the full group.

By decomposing the classical string with magnetic charge $p$ into irreducible representations of $\mathrm{U}(1)$, as in sect. 3, we find states $|q, p\rangle$ with electric charge $q$, where $q$ is any integer. Re-analyzing the charge transfer process, we find that, when loop 2 winds through loop 1, the charge assignments change according to

$$
\left|q_{1}, p_{1} ; q_{2}, p_{2}\right\rangle \rightarrow\left|q_{1}+2 q_{2}, p_{1}+2 p_{2} ;-q_{2},-p_{2}\right\rangle
$$

Naturally, both magnetic charge and electric charge are exchanged.

We will comment briefly on how the analysis is modified when the vacuum $\theta$-angle is non-zero. The non-vanishing vacuum angle alters the $\mathrm{U}(1)$ transforma- 
tion properties of states with non-zero magnetic charge, so that eq. (3.5) is replaced by [17]

$$
U\left(\mathrm{e}^{i \omega Q}\right)|q, p\rangle=\exp [i \omega(q+(\theta / 2 \pi) p)]|q, p\rangle
$$

where $Q$ is the $\mathrm{U}(1)$ generator, and $q$ is the charge of the state defined in terms of the electric flux through the surface at spatial infinity. Thus, for magnetically charged string loops, as for all magnetically charged objects, the charge spectrum is displaced away from the integers by $-\theta p / 2 \pi$. But otherwise, the discussion of electric and magnetic charge transfer is not altered; in particular, eq. (4.15) still applies.

\subsection{LINKED LOOPS}

The homomorphism defined in eq. (4.2) assigns a magnetic charge to any region whose boundary is homeomorphic to $S^{2}$. But if two string loops link, the magnetic charge on each individual loop is not well defined in general. Only the total magnetic charge of the two loops can be defined. The magnetic field of a pair of linked loops has some interesting properties that we will briefly discuss.

In general, two non-abelian string loops can link only if the commutator of their fluxes is in the connected component of the unbroken group *. This feature is a consequence of the "entanglement" phenomenon. Suppose that a string loop with flux $h_{1}$ and a string loop with flux $h_{2}$ cross each other, and become linked. After they cross, a flux $h_{1} h_{2} h_{1}^{-1} h_{2}^{-1}$ must flow from one loop to the other [14,18-21]. If this commutator is not in the connected component of $\mathrm{H}$, then the commutator flux is itself confined to a stable string. Thus, the two loops must be connected by a segment of string that carries the commutator flux. On the other hand, if the commutator is in the connected component of $\mathrm{H}$, then the commutator flux is unconfined, and the flux will spread out uniformly over the $h_{1}$ and $h_{2}$ loops. The linked loops will have a long-range magnetic dipole field, though the total magnetic charge of the linked pair is zero.

In the case of the Alice string, consider two linked loops that carry flux $X \mathrm{e}^{i \theta_{1} Q}$ and $X \mathrm{e}^{i \theta_{2} Q}$, respectively. The commutator flux $\mathrm{e}^{i 2\left(\theta_{2}-\theta_{1}\right) Q}$ is in $\mathrm{H}_{\mathrm{c}}$, so that linking is allowed. The strength of the dipole field is proportional to $\theta_{2}-\theta_{1}(\bmod 2 \pi)$. If we fix the positions of the loops and specify initial values for $\theta_{1}$ and $\theta_{2}$, then, since the dipole field costs magnetostatic energy, the angle $\theta_{2}-\theta_{1}$ will oscillate and the dipole field will become time dependent. These oscillations will cause emission of radiation, and $\theta_{2}-\theta_{1}$ will decay, eventually approaching zero.

* We thank Tom Imbo for a helpful discussion about this. 


\section{Concluding remarks}

\subsection{TWISTED STRINGS AND POINTLIKE MONOPOLES}

In any model in which a connected gauge group $\mathrm{G}$ breaks to a group $\mathrm{H}$ that has a disconnected component, there will be topologically stable strings. If, in addition, $H$ contains non-contractible closed paths (and $\mathrm{G}$ is simply connected), then a string loop can have a topological twist. We have seen that the topological charge that characterizes the twist on the string is precisely the $\mathrm{H}$ magnetic charge. Indeed, magnetically charged string loops exist in any model that contains both strings and magnetic monopoles.

But it is not guaranteed that the twist will be stable. Even if the loop is held in place so that it does not contract, it may be energetically favorable for the loop to untwist and spit out a pointlike magnetic monopole. Or conceivably, the monopole may prefer to stick to the string. In other words, the twisting may be localized in the vicinity of a point on the string loop, so that the pointlike magnetic monopole is a "bead" that can slide along the string *.

Unless there is a symmetry to enforce a degeneracy, vortices with different values of the flux are generically expected to have different masses. Correspondingly, twisting the flux on a string loop will generically increase the potential energy stored in the core of the string. Thus, the flux will prefer to stay near the value that minimizes the core energy, and will twist suddenly inside the "bead".

A gradual twist on a string is expected to be stable, then, only if the flux on the string stays in the orbit of the unbroken symmetry group of the theory. This symmetry cannot be a global symmetry. The identity component of the global symmetry group must commute with the gauge group, so the action of a global symmetry cannot rotate the flux continuously. We conclude that, barring accidental degeneracy, stable twists can reside only on Alice-like strings - that is, the unbroken gauge group must act non-trivially on the flux of the string.

Even for an Alice string, there may be values of the magnetic charge that cannot be carried by the string. To illustrate this point, consider a model with a hierarchy of symmetry breakdown

$$
\mathrm{SU}(3) \stackrel{v_{1}}{\longrightarrow} \mathrm{SO}(3) \stackrel{v_{2}}{\longrightarrow} \mathrm{O}(2), \quad v_{1} \gg v_{2} .
$$

At the large symmetry-breaking mass scale $v_{1}$, the gauge group $\mathrm{SU}(3)$ is broken to its $S O(3)$ subgroup, embedded so that the 3 of SU(3) transforms as the 3 of SO(3). At the much smaller scale $v_{2}$, Alice symmetry breaking occurs, to the unbroken group $\mathrm{O}(2)$. Thus, this model contains Alice strings with tension of order $v_{2}^{2}$, and

* Here, in contrast to the beads considered in ref. [22], the magnetic charge carried by the monopole is unconfined. 
magnetic monopoles with mass of order $v_{2} / e$, where $e$ is the gauge coupling. The properties of these strings and monopoles can be accurately described in an effective field theory, with the very-short-distance physics at scale $v_{1}$ integrated out. This effective theory is essentially the model discussed in sect. 2 .

The new feature of this model, though, is that, because $\mathrm{SO}(3)$ is embedded in SU(3), there is a heavy monopole with mass of order $v_{1} / e$, that carries half the magnetic charge of a light monopole. (The magnetic charge of a light monopole is characterized by a non-contractible loop in U(1) that begins at the identity and ends at a $4 \pi$ rotation, a loop that is contractible in SO(3). The magnetic charge of the heavy monopole is characterized by a loop that ends at a $2 \pi$ rotation. This loop is non-contractible in SO(3), but can be contracted in SU(3).) Therefore, the magnetic charge carried by a twisted loop of Alice string is actually an even multiple of the magnetic charge quantum in this model. Correspondingly, since all representations of $\mathrm{SU}(3)$ are integer spin representations of $\mathrm{SO}(3)$, the string can carry the electric charge quantum.

As our general discussion in sect. 4.1 indicates, it is in a sense possible to construct string configurations with a "half twist" that carry the magnetic charge quantum. Outside this string, the Higgs field takes values in the $S U(3) / O(2)$ vacuum manifold. But unlike the case of a string with a full twist, the Higgs field does not stay within the $\mathrm{SO}(3) / \mathrm{O}(2)$ submanifold. Heavy Higgs fields (and gauge fields) are necessarily excited in the core of the string. It is energetically favorable for the half twist to shrink to a bead, of to form a heavy monopole that is ejected from the loop. (Which of these scenarios is realized depends on the details of the model.) The stable twists on the string can be correctly analyzed using the effective theory, in which the higher scale of symmetry breakdown is disregarded.

Even if $v_{1}$ and $v_{2}$ are of the same order of magnitude, so that there is no large symmetry breaking hierarchy, the half twist is expected to be unstable in this model. For the Alice string with a stable twist, the "vortices" that are associated with various slices through the string are related by a symmetry, and so are degenerate. There is no symmetry to enforce such a degeneracy for the string with a half twist. So introducing a half twist generically increases the core energy, which destabilizes the twist.

\subsection{STABLE LOOPS}

A loop with Cheshire magnetic charge will have Coulomb energy of order

$$
E_{\text {Coulomb }} \sim p^{2} / e^{2} R
$$

where $p$ is the charge in units of the Dirac magnetic charge, $e$ is the gauge coupling, and $R$ is the size of the loop. When the charge $p$ is large, classically-stable string loop configurations can be constructed, such that the Coulomb potential 
energy prevents the loop from collapsing. If the charge is Cheshire charge, then the size $R$ and mass $m$ of a stable loop are, in order of magnitude,

$$
R \sim(p / e) \kappa^{-1 / 2}, \quad m \sim(p / e) \kappa^{1 / 2},
$$

where $\kappa$ is the string tension. Though classically stable, these string loops are not expected to be absolutely stable; they will emit elementary monopoles via a quantum tunneling process, assuming that the emission is kinematically allowed.

\subsection{COSMOLOGICAL ALICE STRINGS}

Because a twisted Alice string carries magnetic charge, we see that a phase transition that produces Alice strings must also produce magnetic monopoles. This observation significantly restricts the role that Alice strings can play in cosmology. If Alice strings ever appeared in the early universe, an unacceptably large abundance of monopoles must have appeared at the same time [23]. Some subsequent mechanism must have reduced the monopole abundance to an acceptable level; we should then ask whether the strings could have survived. For example, if the phase transition that produced the monopoles were followed by an inflationary epoch, the monopole abundance would have been drastically reduced. But, of course, the inflation also would have made Alice strings extremely scarce.

One caveat should be mentioned. The remark in the previous paragraph applies to any model such that the unbroken gauge group $H$ contains a U(1) factor and a charge conjugation operator that acts non-trivially on the U(1) generator $Q$. But it need not apply to models that exhibit the generalized Alice-like behavior considered at the end of sect. 2. In particular, a model with the unbroken group $\mathrm{H}=\left[\mathrm{SU}(2)_{1} \times \mathrm{SU}(2)_{2}\right] \times_{\text {S.D. }} \mathbb{Z}_{2}$ contains generalized Alice strings. But since $\mathrm{H}$ is simply connected, this model contains no magnetic monopoles.

We should note another generic feature of realistic models that contain Alice strings. Charge conjugation is not a manifest exact symmetry in nature. So if charge conjugation is a local symmetry (which must be exact), it must be spontaneously broken. In a realistic model, then, Alice strings (at low temperature) are boundaries of charge-conjugation domain walls. If Alice strings were ever present in the early universe, they eventually became confined by walls. Soon after the appearance of the walls, the string network broke up and disappeared [6,24,25].

\subsection{GLOBAL STRINGS AND MONOPOLES}

Finally, we remark that the discussion of magnetic charge transfer in sect. 4 also applies to the line and point defects that arise when a global symmetry group $G$ becomes spontaneously broken to a subgroup H. (Such defects can occur in certain condensed matter systems, such as nematic liquid crystals [19].) By a standard 
argument $[6,16,19]$, the magnetic charge, classified by $\pi_{1}[\mathrm{H}]$, is seen to be equivalent to the topological charge of the order parameter $\Phi$, classified by $\pi_{2}[\mathrm{G} / \mathrm{H}]$ (assuming that $\mathrm{G}$ is simply connected). Thus, our previous analysis applies, without modification, to the transfer of topological charge between a "global monopole" and a "global Alice string."

Recently, Brekke et al. [26] have independently investigated the properties of magnetically charged Alice strings.

We thank Mark Alford, Katherine Benson, Rick Davis, Tom Imbo, Kai-Ming Lee, John March-Russell, Sandip Trivedi and Piljin Yi for interesting discussions.

\section{References}

[1] A.S. Schwarz, Nucl. Phys. B208 (1982) 141;

A.S. Schwarz and Y.S. Tyupkin, Nucl. Phys. B209 (1982) 427

[2] J. Kiskis, Phys. Rev. D17 (1978) 3196

[3] S. Coleman and P. Ginsparg, unpublished (1982)

[4] M.G. Alford, K. Benson, S. Coleman, J. March-Russell and F. Wilczek, Phys. Rev. Lett. 64 (1990) 1632; [Erratum: 65 (1990) 668]

[5] J. Preskill and L. Krauss, Nucl. Phys. B34I (1990) 50

[6] J. Preskill, "Vortices and Monopoles," in Architecture of the fundamental interactions at short distances, ed. P. Ramond and R. Stora (North Holland, Amsterdam, 1987)

[7] M. Alford, K. Benson, S. Coleman, J. March-Russell and F. Wilczek, Nucl. Phys. B349 (1991) 414

[8] A. Balachandran, F. Lizzi and V. Rodgers, Phys. Rev. Lett. 52 (1984) 1818

[9] P. Nelson and A. Manohar, Phys. Rev. Lett. 50 (1983) 943;

A. Balachandran, G. Marmo, N. Mukunda, J. Nilsson, E. Sudarshan and F. Zaccaria, Phys. Rev. Lett. 50 (1983) 1553;

P. Nelson and S. Coleman, Nucl. Phys. B237 (1984) 1

[10] M. Bucher, On the theory of non-abelian vortices and cosmic strings, Ph.D. Thesis (Caltech, 1990)

[11] E. Witten, Nucl. Phys. B249 (1985) 557

[12] F. Wilczek and Y.-S. Wu, Phys. Rev. Lett. 65 (1990) 13

[13] M. Bucher, Nucl. Phys. B350 (1991) 163

[14] M. Alford, K.-M. Lee, J. March-Russell and J. Preskill, Nucl. Phys. B384 (1992) 251

[15] E. Lubkin, Ann. Phys. (N.Y.) 23 (1963) 233

[16] S. Coleman, The magnetic monopole fifty years later, in The unity of the fundamental interactions, ed. A. Zichichi (Plenum, New York, 1983)

[17] E. Witten, Phys. Lett. B86 (1979) 283;

F. Wilczek, Phys. Rev. Lett. 48 (1982) 1146

[18] V. Poénaru and G. Toulouse, J. Phys. (Paris) 38 (1977) 887

[19] N.D. Mermin, Rev. Mod. Phys. 51 (1979) 591

[20] F. Bais, Nucl. Phys. B170 (1980) 32

[21] T.W.B. Kibble, Phys. Rep. 67 (1980) 183

[22] M. Hindmarsh and T.W.B. Kibble, Phys. Rev. Lett. 55 (1985) 2398

[23] J. Preskill, Phys. Rev. Lett. 43 (1979) 1365

[24] T.W.B. Kibble, G. Lazarides and Q. Shafi, Phys. Rev. D26 (1982) 435

[25] A. Vilenkin and A.E. Everett, Phys. Rev. Lett. 48 (1982) 1867

[26] L. Brekke, W. Fischler and T. Imbo, Phys. Rev. Lett. 67 (1991) 3643 